\title{
Classical Spin-Orbit Coupling and Periastron Advance in PSR J0045-7319
}

\author{
Victoria M. Kaspi \\ $I P A C /$ Caltech $/ J P L$
}

\begin{abstract}
Radio timing observations of the SMC binary pulsar PSR J0045-7319 show, for the first time in a binary pulsar, that classical spin-orbit coupling and periastron advance are occurring. From the observations, the angle $\theta$ between the $B$ star spin axis and the orbital angular momentum is constrained to be $25^{\circ}<\theta<41^{\circ}$ (Kaspi et al. 1996b). Under the conventional assumption that the pre-supernova angular momenta were aligned, this provides direct evidence for an asymmetric supernova explosion. In addition, a large, unexpected orbital decay is observed in the system.
\end{abstract}

\section{Introduction}

PSR J0045-7319 is a 0.926 -s radio pulsar in an eccentric 51-day binary in the SMC, as determined from pulse timing measurements at the Parkes telescope (Kaspi et. al. 1994). Its companion is a main-sequence B star, making this the second known pulsar/B star binary after PSR B1259-63 (see Johnston, this volume). Doppler shifts of the companion's spectral lines have been detected at the expected orbital phase (Bell et al. 1995). The amplitude of the B star's radial velocity variation determines the mass ratio in the system to be $6.3 \pm 1.2$. For a $1.4 \mathrm{M}_{\odot}$ neutron star, the B star's mass is $(8.8 \pm 1.8) \mathrm{M}_{\odot}$, implying that the orbital angular momentum vector is inclined by $44^{\circ} \pm 3^{\circ}$ with respect to the line of sight.

From the known system parameters, at periastron, the pulsar's distance from its companion is $(3.7 \pm 0.5) R_{c}$, while at apastron the separation is ( $35 \pm$ $5) R_{\mathrm{c}}$, where the companion radius, $R_{\mathrm{c}}=(6.4 \pm 0.7) R_{\odot}$, is known from the optical observations (Bell et al. 1995). In spite of the close periastron approach, no variations in the electron column density have been observed with orbital phase, placing a tight limit on mass loss from the B star (Kaspi, Tauris \& Manchester 1996). Nevertheless, significant deviations from a Keplerian orbit have been detected (Kaspi et al. 1996a). Lai, Bildsten \& Kaspi (1995) showed that the deviations were qualitatively consistent with classical spin-orbit coupling and periastron advance due to the B star's rotationally-induced quadrupole moment, which is expected to be large because of its rapid rotation. Spin-orbit coupling, observed as a precession of the orbital plane, requires the B star and orbital angular momenta to be misaligned. 


\section{Classical Spin-orbit Coupling and Periastron Advance}

Kaspi et al. (1996b) show that the timing residuals for PSR J0045-7319 are welldescribed by a model including standard astrometric, spin, and Keplerian orbital parameters, in addition to a periastron advance $\dot{\omega}=\left(0^{\circ} .0259 \pm 0^{\circ} .0005\right) \mathrm{yr}^{-1}$, a. varying projected semi-major axis $\dot{x} \equiv \mathrm{d}(a \sin i) / \mathrm{dt}=(1.17 \pm 0.02)$ It s s${ }^{-1}$ (interpreted as a varying orbital inclination angle $i$ ), as well as an orbital period derivative $\dot{P}_{b}=(-3.03 \pm 0.09)$. A cubic term remains in the residuals as well, but is consistent with "timing noise," seen in most young pulsars (Arzoumanian et al. 1994). The observed $\dot{\omega}$ and $\dot{x}$ constrain $\theta$, the angle between the $\mathrm{B}$ star and orbital angular momenta, to be $25^{\circ}<\theta<41^{\circ}$. Retrograde $\mathrm{B}$ star rotation is not ruled out by these observations. This represents the first proof of misalignment in a massive neutron star binary, and the first detection of classical spin-orbit coupling outside the Solar System. Under the conventional assumption that the pre-supernova angular momenta were aligned, the non-zero $\theta$ implies that the supernova explosion that gave birth to the pulsar was asymmetric, but does not strongly constrain the magnitude of the kick imparted to the neutron star, since the progenitor binary parameters are not known. Nevertheless, the kick had to have been at least $\sim 50 \mathrm{~km} \mathrm{~s}^{-1}$, and was probably much larger.

The observed orbital decay time scale is only $0.5 \mathrm{Myr}$, less than the expected evolution time scale of the B star, and less than the characteristic age of the pulsar ( $3 \mathrm{Myr}$ ). The orbital decay is much larger than has been predicted (e.g. Kumar et al. 1995). Lai (1996) argues that if the B star's rotation is retrograde, the dynamical tidal dissipation from coupling between low-order g-modes in the B star and the pulsar's orbit is large enough to account for the orbital decay. In his model, the binary system can be no older than $\sim 1.4 \mathrm{Myr}$, smaller than the characteristic age of the pulsar. This, he suggests, implies that the initial spin period of the pulsar was comparable to its current spin period.

We thank MB, JFB, DL, RNM, BWS, and TT for collaborations and discussions, and CIT, HF, IPAC, JPL, NASA, and STScI for support.

\section{References}

Arzoumanian, Z., Nice, D.J., Taylor, J.H. \& Thorsett, S.E., 1994, ApJ, 422, 671

Bell, J. F., Bessell, M. S., Stappers, B. W., Bailes, M. \& Kaspi, V. M., 1994, ApJ, 447, L117

Kaspi, V. M., Johnston, S., Bell, J. F., Manchester, R. N., Bailes, M., Bessell, M., Lyne, A. G. \& D'Amico, N., 1994, ApJ, 423, L43

Kaspi, V. M., Manchester, R. N., Bailes, M., Bell, J. F., 1996a, in Compact Stars in Binaries, IAU Symp. 165, Kluwer, Dordrecht, 271

Kaspi, V. M., Tauris, T. \& Manchester, R. N., 1996, ApJ, 459, 717

Kaspi, V. M., Bailes, M., Manchester, R. N., Stappers, B. W., Bell, J. F., 1996b, Nature, submitted

Kumar, P., Ao, C. O. \& Quataert, E. J., 1995, ApJ, 449, 294

Lai, D., Bildsten, L. \& Kaspi, V. M., 1995, ApJ, 452, 819

Lai, D., 1996, ApJ, submitted 